\section{$\underset{\substack{\text { hommes } \\ \text { \& migrations }}}{ }$}

\section{Hommes \& migrations}

Revue française de référence sur les dynamiques

migratoires

1289 | 2011

Les frontières du sport

\title{
Immigration polonaise et pratique sportive en milieu de grande industrie
}

Le cas du bassin de Montceau-les-Mines dans l'entre-deux-guerres

\section{Karen Bretin Maffiuletti}

\section{(2) OpenEdition \\ Journals}

Édition électronique

URL : http://journals.openedition.org/hommesmigrations/793

DOI : 10.4000/hommesmigrations.793

ISSN : 2262-3353

Éditeur

Musée national de l'histoire de l'immigration

Édition imprimée

Date de publication : 1 janvier 2011

Pagination : 38-47

ISSN : 1142-852X

\section{Référence électronique}

Karen Bretin Maffiuletti, «Immigration polonaise et pratique sportive en milieu de grande industrie », Hommes \& migrations [En ligne], 1289 | 2011, mis en ligne le 01 janvier 2013, consulté le 01 mai 2019. URL : http://journals.openedition.org/hommesmigrations/793 ; DOI : 10.4000/

hommesmigrations.793 


\section{Immigration polonaise et pratique sportive en milieu de grande industrie}

\section{Le cas du bassin de Montceau-les-Mines dans l'entre-deux-guerres}

Par Karen Bretin Maffiuletti, maître de conférences en histoire du sport, laboratoire de socio-psychologie et management du sport EA 4180, université de Bourgogne

Ouvriers polonais de la navigation sur le canal à Montceaux-les-Mines, 1933. Archive privée extraite de l'ouvrage d’Anna Nawrocki, La Bourgogne et la Pologne à travers les siècles, Neuilly-lès-Dijon, Murmure, $2002 \odot$ D.R.

Ancienne ville minière, Montceau-les-Mines compte une importante communauté polonaise. À leur arrivée en masse au début des années vingt, les migrants polonais créent leurs propres associations de loisirs dans un territoire dominé par la pratique du cyclisme et de la gymnastique. Loin de témoigner d'un engouement partagé avec les Montcelliens pour le sport, la vitalité du réseau associatif polonais indique la persistance d'une frontière culturelle. À contre-pied des supposées vertus intégratrices du sport, la pratique des loisirs à Montceau-les-Mines écrit l'histoire d'une non-rencontre. 
Depuis quelques années, les chercheurs questionnent l'idée selon laquelle le sport a constitué dans l'histoire un moyen privilégié d'intégration des Français "venus d'ailleurs ${ }^{(1)}$ ", et notamment des populations immigrées. Les ambivalences du sport, tantôt vecteur de lien social, tantôt générateur d'exclusion, sont pointées, de même que le caractère exceptionnel de l'accès au statut de champion, au regard de la masse des prétendants à la gloire sportive ${ }^{(2)}$. Il apparaît également que l'engagement des immigrés dans le mouvement sportif, s'il favorise les contacts avec les milieux ouvriers "français", s'accompagne aussi d'une forme d'“exploitation" des étrangers par les catégories dominantes impliquées dans le monde du sport ${ }^{(3)}$. Au final, c'est bien le mythe d'un sport intégrateur par essence qui est dénoncé.

Toutefois, si les rapports entre sport et immigration sont aujourd'hui couramment discutés, la pratique des activités physiques reste peu mobilisée en tant qu'analyseur, à un niveau plus global, des trajectoires migratoires. La présente contribution s'inscrit dans cette perspective. Elle est liée à un projet de recherche visant à définir la place du sport et des loisirs dans le quotidien de populations immigrées, et à mesurer l'impact de ces pratiques sur les parcours et sur les interactions avec la société française et les Français "de souche". Ainsi, l'ambition est bien de penser le sport comme une dimension parmi d'autres de l'existence de populations étrangères et, selon le vœu formulé par certains spécialistes ${ }^{(4)}$, de se détourner du sport et des sportifs de bon niveau pour s'intéresser à des immigrés et des pratiques de loisirs ordinaires. Le cadre d'observation, nécessairement restreint compte tenu des objectifs fixés, est celui du bassin minier de Montceau-les-Mines, en Saône-et-Loire. Ce milieu de grande industrie est animé, au fil du temps, par des travailleurs de multiples nationalités, mais c'est bien la présence polonaise qui est ici la plus nombreuse et qui marque le plus profondément l'histoire de la région. Les investigations portent donc naturellement sur cette population. Enfin, la période retenue s'étend du début des années vingt à la veille de la Seconde Guerre mondiale. Elle répond au souhait d'analyser la rencontre entre une première génération de travailleurs étrangers et la réalité montcellienne, en particulier au plan de la vie sportive. Les premiers résultats d'une telle enquête sont dévoilés ici.

Il s'agit d'analyser la participation polonaise au mouvement sportif du bassin minier et, plus largement, d'amorcer une étude des comportements de loisirs de cette population. On débute par une présentation plus détaillée de la région montcellienne et des conditions de l'immigration polonaise dans cet espace. L'engagement des Polonais dans le mouvement sportif régional est ensuite appréhendé essentiellement par le biais d'une histoire institutionnelle. L'interprétation des faits mis en évidence signale, pour finir, plusieurs pistes de recherche pour des investigations complémentaires. 


\section{Le bassin minier de Montceau-les-Mines : grande industrie et immigration polonaise}

L'histoire industrielle du bassin minier de Saône-et-Loire débute dès les années dixhuit cent trente, avec la découverte de gisements de charbon importants et la formation en 1833 de la Compagnie des houillères de Blanzy. Celle-ci se développe rapidement. Avec les Établissements métallurgiques du Creusot, dont elle accompagne et rend possible le succès à l'échelle internationale, l'entreprise d'extraction minière incarne depuis le milieu du XIX' siècle et jusqu'aux années soixante la figure de proue de l'industrie bourguignonne. La cité de Montceau-les-Mines naît en 1856 autour du siège de la Compagnie et constitue rapidement le cceur du bassin minier. Elle se confond avec les petites villes limitrophes de Blanzy, Saint-Vallier et Sanvignes-lesMines pour former un tout, retenu comme cadre d'observation : la Compagnie des houillères fait vivre le territoire dans son ensemble et emploie la majorité de ses habitants, emportés dans une histoire commune.

Les premiers ouvriers mineurs de Montceau et des cités voisines sont essentiellement d'anciens cultivateurs et vignerons, issus des campagnes environnantes, mais les besoins de main-d'cuvre sans cesse croissants drainent vers le bassin minier des travailleurs originaires d'autres régions de France, avant et pendant la Première Guerre mondiale. L'arrivée massive d'ouvriers étrangers s'effectue, comme partout ailleurs en France ${ }^{(5)}$, dans l'entre-deux-guerres. Aux Houillères, les effectifs des immigrés passent de 445 en 1920

\section{Entre les années vingt et les années trente, la région montcellienne compte en moyenne 7000 Polonais, qui représentent 70 à $80 \%$ des étrangers de cet espace et $20 \%$ environ de la population totale.} à 3000 sur 9476 salariés en 1922. En janvier 1931, les ouvriers étrangers représentent plus de $40 \%$ de l'ensemble du personnel ${ }^{(6)}$. L'arrivée des travailleurs polonais et de leurs familles s'effectue dès le début des années vingt. Entre les années vingt et les années trente, la région montcellienne compte en moyenne 7000 Polonais, qui représentent

70 à $80 \%$ des étrangers de cet espace et $20 \%$ environ de la population totale ${ }^{(7)}$. Linstallation de cette population dans la région suit la même temporalité que le mouvement d'immigration polonaise en France, puisque celui-ci débute à la fin de la Première Guerre mondiale (une convention franco-polonaise, signée le 3 septembre 1919, officialise le processus d'accueil en France de la main-d'ceuvre polonaise) et se prolonge jusqu'à la crise économique des années trente ${ }^{(8)}$. Par ailleurs, de par les effectifs absorbés, le bassin minier de Saône-et-Loire constitue bien, après les départements du Nord et du Pas-de-Calais, l'un des principaux foyers d'implantation. 
Les migrants polonais arrivent pour beaucoup directement de leur pays natal, par convois spéciaux de chemin de fer. Lorsqu'ils rejoignent la région montcellienne, ils sont pris en charge par la Compagnie des houillères, qui fournit un logement en cité ouvrière et quelques meubles. Le quotidien de ces familles s'organise naturellement autour du travail à la mine : huit heures par jour et six jours par semaine. Plus souvent que les Français, les ouvriers polonais s'échinent "au fond", dans les tâches les plus éprouvantes ${ }^{(9)}$. Toutefois, en marge de cette vie de mineur, marquée par la dureté du travail et la fatigue qu'elle engendre, des activités de loisirs (notamment sportives) demeurent. Lobservation de ces pratiques signale alors des pistes nouvelles pour définir les modalités de la rencontre entre Polonais et Montcelliens et pour analyser une situation migratoire particulière.

\section{Séparation des travailleurs polonais et montcelliens dans le loisir sportif}

Le bassin minier de Saône-et-Loire est une terre de forte tradition sportive. Dès la fin du XIX ${ }^{\mathrm{e}}$ siècle, plusieurs sociétés d'activités physiques sont formées à Montceau-lesMines. Elles organisent la pratique du tir, du cyclisme, des joutes, de la natation et de la gymnastique. Plus tard, le mouvement sportif s'enrichit notamment de clubs de boxe, de tennis et de football-rugby, ainsi que de nouvelles sociétés de cyclisme et de gymnastique. Durant les années vingt et les années trente, on peut estimer à une trentaine le nombre des structures, le plus souvent affinitaires (patronales, ouvrières ou catholiques), qui se partagent (ou se disputent) le territoire et les sportifs de Montceau-les-Mines et des villes voisines. Ces groupements dynamiques, bien connus et largement investis par les habitants, notamment les ouvriers mineurs, jouent un rôle important dans la vie des localités et dans le quotidien de la population.

À l'arrivée des migrants polonais, un réseau d'associations assez dense, et offrant des formes de pratiques variées, est donc susceptible d'encadrer leurs loisirs sportifs. Pourtant, aux origines de la vague migratoire, comme durant les années trente, tout indique que cette population nouvelle s'engage très peu dans les structures déjà en place. On ne repère tout d'abord pratiquement aucun patronyme polonais dans les listes de membres des associations sportives de Montceau, Blanzy, Sanvignes et SaintVallier, consultables aux archives départementales de Saône-et-Loire ${ }^{(10)}$. Les témoignages d'anciens pratiquants puis dirigeants vont également dans ce sens. Ces derniers témoignent en effet de l'absence de travailleurs ou de jeunes Polonais parmi leurs camarades d'entraînement dans les années trente. Ils affirment également avoir le sentiment qu'il en allait de même, d'une manière générale, dans l'ensemble des structures sportives du bassin minier ${ }^{(11)}$. Surtout, l'analyse des rapports fournis par les 
autorités du bassin minier dans le cadre d'une politique de surveillance des étrangers, prégnante dès le début des années trente et qui se renforce au cours de la décennie, permet la mise en évidence de nombreuses associations sportives polonaises, créées et exclusivement fréquentées par les migrants ${ }^{(12)}$.

\section{Les sociétés sportives: un marqueur des origines}

L'Union sportive Polonia et une société baptisée "Union polonaise de tir de France" sont respectivement créés en 1928 et 1931 dans les cités ouvrières des Gautherets à Saint-Vallier et de La Saule à Montceau-les-Mines. Elles organisent les pratiques du football, du tir et de la gymnastique. De même, Sanvignes accueille une Société des sokols (gymnastique, mais aussi athlétisme, football, cyclisme, lutte, boxe) et l'association les Amis des boy-scouts (activités diverses), signalées dès 1930. Une deuxième association de football, le Club sportif Orion, est fondée à Montceau-lesMines en 1933. En 1937, neuf nouvelles structures sont signalées, dont trois sociétés de tir et deux associations illustrant l'extension géographique d'organisations déjà existantes. Après Sanvignes, Montceau se dote également de sa Société des sokols et d'un club de boy-scouts. Enfin, à la veille de la Seconde Guerre mondiale, le bassin minier compte au moins une vingtaine de sociétés sportives polonaises, établies dans une dizaine de cités ouvrières différentes, à Montceau, Sanvignes et Saint-Vallier. Aux premières sociétés, toujours actives, se sont ajoutés des clubs de tir (décidément très présents) et plusieurs sections de l'Union des éclaireurs polonais en France.

De telles observations suggèrent bien que la vie sportive des immigrés polonais et de leurs familles se situe ailleurs que dans les associations du cru. Enfin, il faut remarquer que les clubs sportifs polonais ne constituent que l'un des aspects d'un réseau d'associations beaucoup plus vaste. Le bassin minier de Montceau compte également, à l'époque, près de soixante-dix sociétés religieuses et/ou culturelles polonaises ${ }^{(13)}$, qui encadrent de la même façon le temps libre des migrants des deux sexes et de tous âges. "Hors travail", la séparation des différentes communautés ouvrières se confirme.

\section{Comprendre une non-rencontre}

L'observation d'une distance séparant la communauté polonaise et les Montcelliens "de souche" dans la pratique sportive et de loisirs peut surprendre les défenseurs de discours convenus sur les vertus intégratrices du sport. Elle est néanmoins cohérente avec les résultats d'enquêtes déjà conduites sur des terrains comparables. Ainsi, Janine 


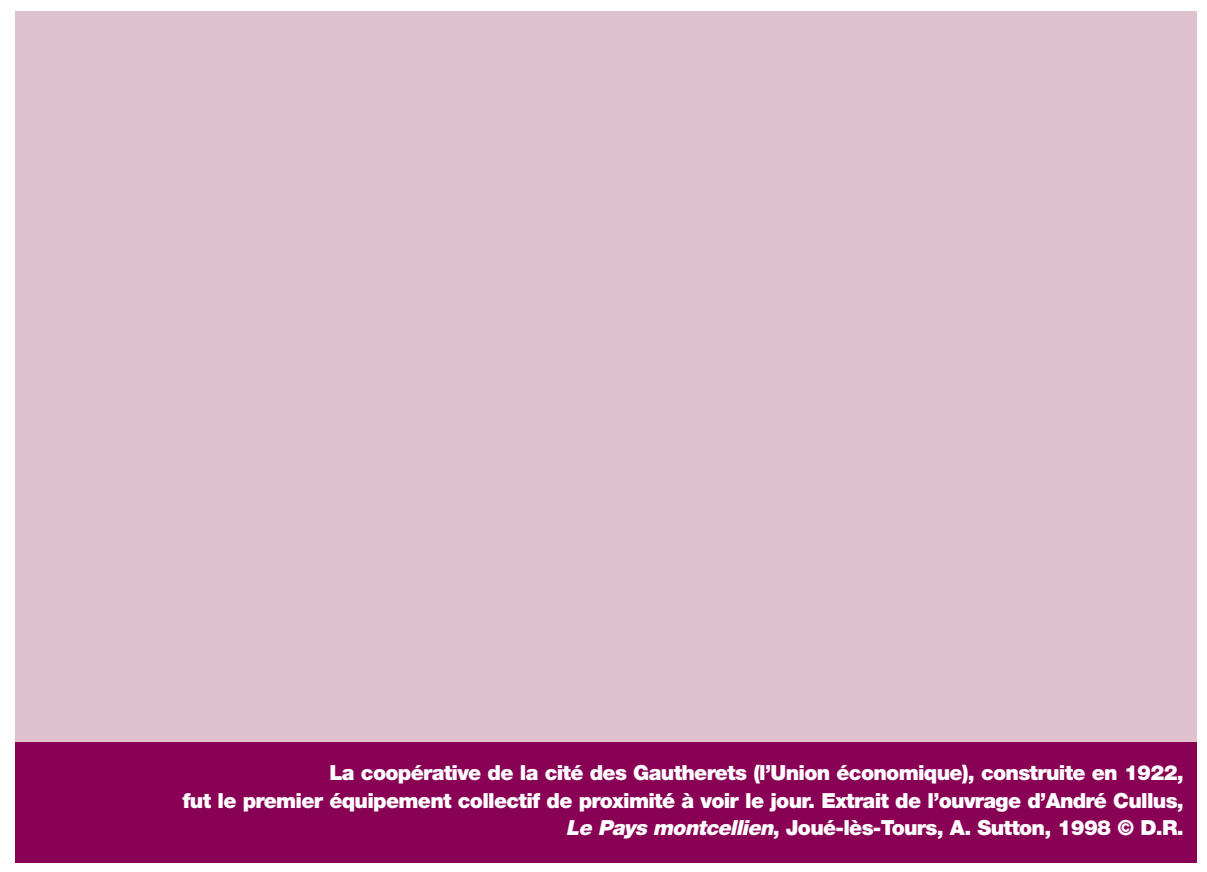

Ponty, Marion Fontaine et dans une moindre mesure Olivier Chovaux notent le faible engagement des premiers immigrés polonais dans le mouvement associatif du Nord ${ }^{(14)}$. Parallèlement, plusieurs spécialistes ont déjà détaillé les caractéristiques du tissu associatif polonais en France, soulignant son dynamisme et une bonne pénétration géographique ${ }^{(15)}$. La densité du réseau associatif polonais de Montceau ne fait donc que répondre à l'importance de ce même réseau à l'échelle nationale. Par ailleurs, les faits mis en évidence dans le bassin montcellien peuvent trouver différentes justifications, qui mêlent étroitement l'histoire de l'immigration polonaise en France et l'histoire (sociale et sportive) de ce territoire.

\section{Les sports et loisirs, marqueurs de l'autonomie des communautés}

La non-rencontre des populations polonaise et montcellienne dans le loisir sportif peut, d'une part, être interprétée comme le signe d'une "frontière" séparant d'une manière plus générale les deux communautés. Ainsi, il est vrai que les travailleurs polonais et leurs familles occupent dans le bassin minier de Saône-et-Loire des espaces très spécifiques. Les quartiers ouvriers de Montceau-les-Mines et des villes voisines, construits au fil du développement de la Compagnie des houillères, selon un 
découpage presque géométrique du territoire, accueillent chacun une population particulière. Pierre-Jacques Derainne évoque un type de logement "enclave", favorisant "une vie collective entre immigrés de même provenance $e^{16) "}$. À Montceau comme ailleurs, on repère ainsi de "Petites Polognes", soit des quartiers où les migrants polonais passent l'essentiel de leur existence et où ils recréent les conditions de vie de leur pays d'origine ${ }^{(17)}$. Si les associations sportives montcelliennes n'attirent pas les Polonais, c'est donc peut-être simplement (comme on l'a déjà observé en d'autres lieux) parce qu'elles ne font pas partie de leur cadre de vie.

Par ailleurs, au-delà d'une distance purement géographique, une frontière culturelle, non moins importante et déterminante des rapports entre les communautés, sépare également les familles originaires de Pologne et celles de la région. Plusieurs auteurs ont déjà suggéré les profondes divergences culturelles entre les migrants polonais et la population des espaces qui les reçoivent ${ }^{(18)}$. Dans le bassin de Montceau, de la même manière, tout oppose les travailleurs polonais et les "régionaux". Quand la vie des migrants est guidée par la foi catholique et leur quotidien scandé par les cérémonies et fêtes religieuses, les mineurs du cru apparaissent fortement marqués par les idées communistes et fondent leur identité sur une appartenance revendiquée à la catégorie des "rouges" anticléricaux, ceux dont les ancêtres "ont brûlé l'église "(19)". Aux origines de la migration polonaise, les deux populations s'ignorent. Dans une minute adressée au ministre de l'Intérieur en 1927, le préfet de Saône-et-Loire observe ainsi que les Polonais "ont tendance à s'isoler et à ne pas entrer en contact régulier avec les éléments

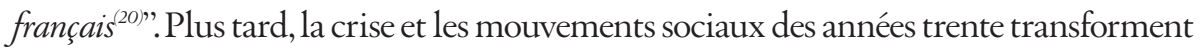
en méfiance, voire en ressentiment une sensation déjà très forte de différence à l'égard de l'autre : les Montcelliens d'origine se font plus durs avec la population polonaise, qui leur rend bien ces mauvais sentiments.

Au final, tout au long de l'entre-deux-guerres, les deux communautés évoluent séparément et il n'est pas surprenant d'effectuer le même constat dans le cadre des loisirs.

\section{Les sports et loisirs comme moyens d'une prise de distance}

Si les loisirs des Polonais et ceux des Montcelliens se déroulent de manière autonome, c'est peut-être parce que ces activités constituent elles-mêmes l'un des moyens de production (ou tout au moins de préservation) de la distance entre les communautés. Les loisirs deviennent alors un "outil" de mise à distance. La séparation des deux populations dans les loisirs n'est plus la conséquence d'un contexte général mais est bel et bien recherchée.

Limmigration polonaise, on le sait, est différente d'autres mouvements migratoires dans le sens où ses acteurs sont pour la grande majorité d'entre eux convaincus d'un 
prochain retour au pays natal. Plus que d'autres, les travailleurs polonais conçoivent leur embauche en France comme une situation provisoire. Dans ces circonstances, ils "résistent [singulièrement] à la francisation ${ }^{211}$ " et manifestent une volonté farouche de cultiver en France les valeurs polonaises, dans l'attente du retour. Dès lors, le mouvement associatif polonais, rapidement structuré en France et dans la région montcellienne, peut être envisagé comme un moyen de préserver l'unité de la communauté polonaise, en évitant dans les loisirs les contacts avec les Français. Ainsi, il est frappant de remarquer que les associations polonaises de Montceau, sportives et plus encore religieuses et culturelles, assurent un parfait maillage du territoire. Le groupement Strzelec, qui organise la pratique du tir, compte ainsi six sections, établies dans des cités ouvrières différentes de Montceau-les-Mines, Saint-Vallier et Sanvignes. De même, pas un quartier n'échappe à la Jeunesse catholique polonaise ou à l'Union des sociétés féminines polonaises, présentes dans toutes les cités ouvrières importantes. Ces créations d'associations, par leur caractère systématique, peuvent attester que les structures n'ont pas pour seule vocation de divertir ou d'instruire les migrants, mais aussi probablement de limiter les échanges avec la société montcellienne et de protéger leur polonité.

\section{Impact des spécificités sportives du territoire}

Enfin, le rapprochement difficile des communautés polonaise et montcellienne dans le temps des loisirs, à la fois signe et ressource d'une frontière entre groupes humains (si l'on en croit les analyses proposées ci-dessus), traduit aussi les spécificités de l'histoire sportive du territoire.

En effet, si la plupart des observations effectuées en Saône-et-Loire, comme les interprétations qui en sont faites, entrent globalement en cohérence avec les résultats et analyses d'autres auteurs, le bassin minier de Montceau-les-Mines n'en présente pas moins, à ce stade de l'enquête, une forme de singularité. Par comparaison avec le nord de la France, la région montcellienne constitue, semble-t-il, un exemple d'autonomie plus complète de la population polonaise pour ce qui est de ses loisirs sportifs. Certes, les Polonais du Nord sont attachés à une vie communautaire, fortement investis dans leurs mouvements associatifs, et ils revendiquent leur polonité, mais il est vrai également qu'une petite partie d'entre eux, contrairement à leurs compatriotes de Montceau, évoluent et se "font un nom" dans des clubs français, et notamment dans de prestigieuses sociétés de football ${ }^{(22)}$. Olivier Chovaux évoque ainsi les joueurs polonais "dont les exploits [dès l'entre-deux-guerres] s'inscrivent au petit panthéon des histoires sportives locales ${ }^{(23)}$. Les chercheurs indiquent alors que ce sont notamment des enjeux sportifs, certainement propres au football, qui favorisent dans ce cas précis le 
rapprochement des communautés : les migrants sont attirés par une potentielle réussite sportive, synonyme de privilèges à la mine et d'ascension sociale, tandis que les dirigeants de clubs, soucieux de constituer les formations les plus performantes possibles, ne peuvent longtemps ignorer les qualités physiques des mineurs polonais. Dès lors, si le bassin de Montceau-les-Mines illustre de manière plus radicale encore les difficultés de la rencontre, sur les terrains de sport, entre les migrants polonais et les milieux ouvriers français, c'est sans doute parce que la tradition sportive de ce territoire s'y prête. À Montceau, terre de gymnastique et de cyclisme, les clubs de football jouent un rôle secondaire, au regard de la place qu'ils occupent dans le nord de la France. L'engagement dans des compétitions de haut niveau et le professionnalisme sont totalement étrangers, à l'époque, aux dirigeants du sport régional, de même que la figure du champion, vedette du football, est certainement peu présente dans l'imaginaire collectif des ouvriers mineurs, "régionaux" ou immigrés.

\section{Conclusion}

Les faits mis en évidence et les interprétations proposées ne constituent que la première étape d'une recherche sur la place et le rôle des sports et loisirs dans le quotidien des immigrés polonais du bassin minier de Saône-et-Loire. Une seconde étape doit permettre, notamment par le biais de l'histoire orale, de confirmer les analyses et de détailler des phénomènes simplement esquissés.

L'histoire sportive de Montceau-les-Mines et des cités voisines traduit le caractère limité, dans l'entre-deux-guerres, des échanges qui s'établissent plus généralement entre les immigrés polonais et les habitants originaires de la région. Elle souligne également que la structure des mouvements associatifs elle-même a pu jouer comme un élément favorisant le maintien et le renforcement d'une distance entre les groupes. Le sport et les loisirs apparaissent ainsi comme les analyseurs pertinents d'une situation migratoire particulière. Une frontière, tout à la fois reflétée et renforcée dans les loisirs, sépare la communauté polonaise de celle des Montcelliens du cru. Une configuration qui correspond mal à l'idée d'une mise à l'écart de la communauté polonaise, dans la mesure où cette distance est pour une part choisie et activement préservée par les Polonais ; mais une configuration qui ne saurait davantage évoquer une forme de communautarisme, tant il est vrai également qu'elle naît d'une profonde indifférence (voire hostilité) des "régionaux".

Dans le cadre des loisirs, comme plus généralement, une frontière existe. Elle satisfait globalement toutes les parties en présence et ce ne sont pas des impératifs sportifs qui, ici et pour cette génération tout au moins, viennent changer la donne. 


\section{Notes}

1. Viet Vincent, Histoire des Français venus d'ailleurs de 1850 à nos jours, Paris, Perrin, 2004.

2. Par exemple, Bancel Nicolas et Blanchard Pascal, "Lintégration par le sport, quelques réflexions autour d'une utopie", in Migrance, n²2, 2003 ; Gastaut Yvan, "Lintégration par le sport : réalités et illusions", in Cahiers français, $\mathrm{n}^{\circ} 320,2004$.

3. Chovaux Olivier, "Le football, un exemple d'intégration de surface dans l'entre-deux-guerres", in Tous gueules noires, Centre historique minier du Nord-Pas-de-Calais, nº, 2004.

4. Gastaut Yvan, "Introduction. Histoire de l'immigration, histoire du sport, perspectives croisées", in Migrance, n²2, 2003, p. 7 .

5. Noiriel Gérard, Le Creuset français. Histoire de limmigration (XIX"XX" siècles), Paris, Seuil, 1988.

6. Derainne Pierre-Jacques, Histoire et mémoire des immigrations en Bourgogne, rapport de recherche, Direction régionale Bourgogne de l'ACSE, Dijon, 2010, p. 84.

7. Ibid., p. 102.

8. Ponty Janine, Polonais méconnus, histoire des travailleurs immigrés en France dans l'entre-deux-guerres, Paris, Publications de La Sorbonne, 1990, p. 35.

9. Derainne Pierre-Jacques, op.cit., p. 78.

10. Archives départementales de Saône-et-Loire (ADSL), M 432 à M 436 : Associations, cercles, sociétés.

11. Entretiens avec M. Audin (pratiquant puis dirigeant de l'Élan gymnique Montceau Bois-du-Verne) et avec M. Besseyrias (pratiquant du Basket club landais puis dirigeant de l'association Basket Montceau Bourgogne), avril 1999 et juin 2006.

12. ADSL, M 1746 et M 1747 : Surveillance des groupements étrangers. Également 1 W 589 et 1 W 590 : Associations étrangères.

13. 33 associations culturelles et 35 associations religieuses différentes, pour la période 1919-1939, sont identifiées. Toutes n'existent probablement pas simultanément. Reste que l'ampleur du mouvement associatif polonais, dans un espace qui demeure géographiquement assez restreint, est frappante.

14. Ponty Janine, op. cit., et "Les Polonais du Nord ou la mémoire des corons", in Autrement, $n^{\circ} 83,1995$. Fontaine Marion, "La polonité face à la sécularisation dans le monde minier lensois", communication au colloque international "Migrations, religions et sécularisation. L'impact des migrations sur les modèles nationaux (Europe et Amérique du Nord)", Paris, 2005 et "Les Polaks et les Sang et Or : une lecture sportive de la relation aux étrangers dans une ville minière", in Terrier Didier et Rainhorn Judith (dir.), Étranges voisins, Rennes, Presses universitaires de Rennes, 2010 ; Chovaux Olivier, "Football minier et immigration. Les limites de l'intégration sportive dans les années trente", in STAPS, n56, 2001.

15. Voir les auteurs déjà cités ainsi que Garçon Gabriel, "La jeunesse polonaise et les organisations catholiques polonaises en France", in Gogolewski Edmond (dir.), La Protection des Polonais en France, problèmes d'intégration et d'assimilation, Villeneuve-d'Ascq, Éditions du conseil scientifique de l'université de Lille-3, 1999.

16. Derainne Pierre-Jacques, art. cit., p. 122.

17. Ponty Janine, "Les Polonais du Nord ou la mémoire des corons", art. cit.

18. Ibid.; Fontaine Marion, "La polonité face à la sécularisation dans le monde minier lensois", art. cit., et "Les Polaks et les Sang et Or : une lecture sportive de la relation aux étrangers dans une ville minière", art.cit.

19. Entretiens avec M. Audin, avril 1999 et juin 2006.

20. Extrait d'une minute du préfet de Saône-et-Loire adressée au ministre de l'Intérieur, janvier 1927, transcrite par Jacques Miroz (L'Immigration polonaise en Bourgogne au XX' siècle, Association culturelle franco-polonaise Warszawa, Dijon, 1979, p. 70).

21. Ponty Janine, Polonais méconnus, histoire des travailleurs immigrés en France dans l'entre-deux-guerres, op. cit., p. 1.

22. Notamment le Racing club de Lens, qui concentre l'attention des chercheurs (Chovaux Olivier, "Football minier et immigration. Les limites de l'intégration sportive dans les années trente", art. cit.; et "Le football, un exemple d'intégration de surface dans l'entre-deux-guerres", art. cit.; Fontaine Marion, "La polonité face à la sécularisation dans le monde minier lensois", art. cit.; et "Les Polaks et les Sang et Or : une lecture sportive de la relation aux étrangers dans une ville minière", art. cit.).

23. Chovaux Olivier, "Le football, un exemple d'intégration de surface dans l'entre-deux-guerres", art. cit., p. 140. 